\title{
Innate immune activation in Alzheimer's disease
}

\author{
Ming-Ming Wang ${ }^{1}$, Dan Miao ${ }^{1}$, Xi-Peng Cao ${ }^{2}$, Lin $\operatorname{Tan}^{1}$, Lan Tan ${ }^{1}$ \\ ${ }^{1}$ Department of Neurology, ${ }^{2}$ Clinical Research Center, Qingdao Municipal Hospital, Qingdao University, Qingdao 266071, China \\ Contributions: (I) Conception and design: L Tan; (II) Administrative support: None; (III) Provision of study materials or patients: None; (IV) \\ Collection and assembly of data: MM Wang, D Miao; (V) Data analysis and interpretation: None; (VI) Manuscript writing: All authors; (VII) Final \\ approval of manuscript: All authors. \\ Correspondence to: Lan Tan. Department of Neurology, Qingdao Municipal Hospital, Qingdao University, Qingdao 266071, China. \\ Email: dr.tanlan@163.com.
}

\begin{abstract}
Alzheimer's disease (AD) is known as the most predominant cause of dementia among the aged people. Previously, two hallmarks of AD pathology including extracellular amyloid- $\beta$ (A $\beta$ ) deposition and neurofibrillary tangles (NFTs) inside neurons have been identified. With a better understanding of this disease, neuroinflammation has been a focus, and as its initial event, innate immune activation plays an indispensable role. In brain, as an endogenous stimulator, extracellular A $\beta$ deposition activates innate immunity through binding to the pattern recognition receptors (PRR), thus leading to the production and release of substantial inflammatory mediators (NO and ROS) and cytokines (IL-1 $\beta$, IL-10, IL-33 and TNF- $\alpha$ ) contributing to the development of AD. Epidemiologic evidence has suggested an affirmative influence of non-steroidal anti-inflammatory drugs (NSAIDs) on delaying the progression of AD. Therefore, blocking the inflammatory process may be an effective way to delay or even cure $\mathrm{AD}$. In this review, we mainly elucidate the mechanism underlying these immune responses in $\mathrm{AD}$ pathogenesis and attempt to seek the therapeutic methods targeting neuroinflammation.
\end{abstract}

Keywords: Alzheimer's disease (AD); amyloid- $\beta(\mathrm{A} \beta)$; innate immunity; neuroinflammation; neuronal loss; tau

Submitted Feb 26, 2018. Accepted for publication Apr 04, 2018.

doi: $10.21037 /$ atm.2018.04.20

View this article at: http://dx.doi.org/10.21037/atm.2018.04.20

\section{Introduction}

At present, there are approximately 40 million people suffering from dementia in the world and most of them are older than 60 years. Worst of all, at least before 2050, this amount is predicted to double every 20 years (1). Alzheimer's disease (AD) is the most predominant cause of dementia among the aged people and it is a complex progressive neurodegenerative disease characterized by extracellular amyloid- $\beta$ (A $\beta$ ) deposition and neurofibrillary tangles (NFTs) inside neurons. Short-term memory failure combined with behavior deficiency marks the beginning of $\mathrm{AD}$. Ultimately patients with this incurable disease will end up dead. Up to now, there is no efficient treatment to cure $\mathrm{AD}$ or halt its progression. Likewise, the biomarkers for early diagnosis seem to be unaccessible and invalid. The good news is that a high reversion rate from mild cognitive impairment (MCI)-namely the predementia stage to normal cognition is up to $24 \%$ (2). Therefore, elucidating the mechanism underlying $\mathrm{AD}$ is really important to seek therapeutic strategies for delaying or preventing this disease. Previously, investigations of pathological mechanism of $\mathrm{AD}$ were driven by the amyloid cascade hypothesis. With a better understanding of this disease, neuroinflammation has become another crucial factor in the development of AD pathology. As a potent stimulator, extracellular $A \beta$ deposition binding to pattern recognition receptors (PRR) on the surface of microglia membrane triggers innate immune activation, thus leading to the production and release of substantial inflammatory cytokines which induces synapse dysfunction, neuronal loss, concomitantly cognitive decline and memory loss (Figure 1). Thus, we offer an overview of immune responses 


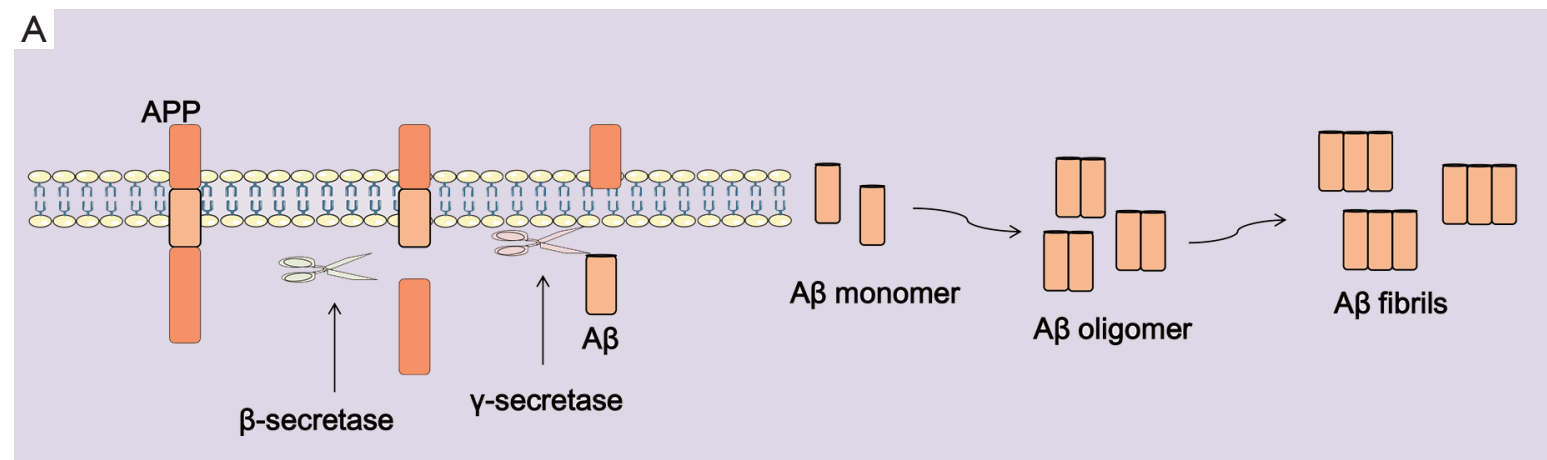

B

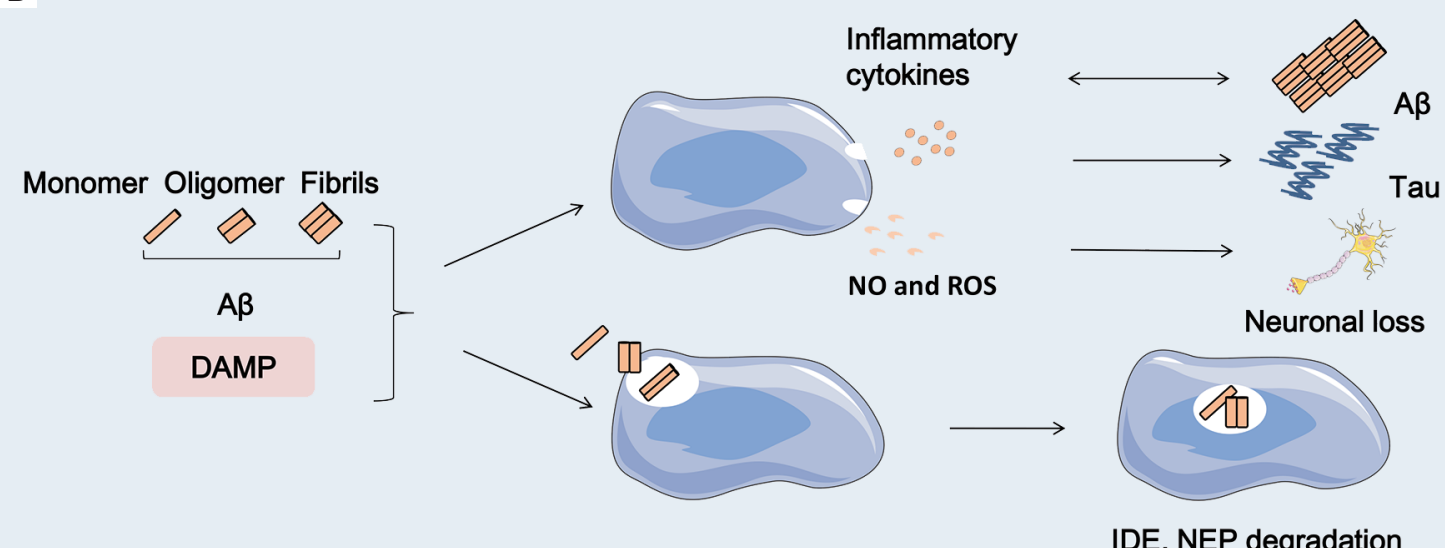

C
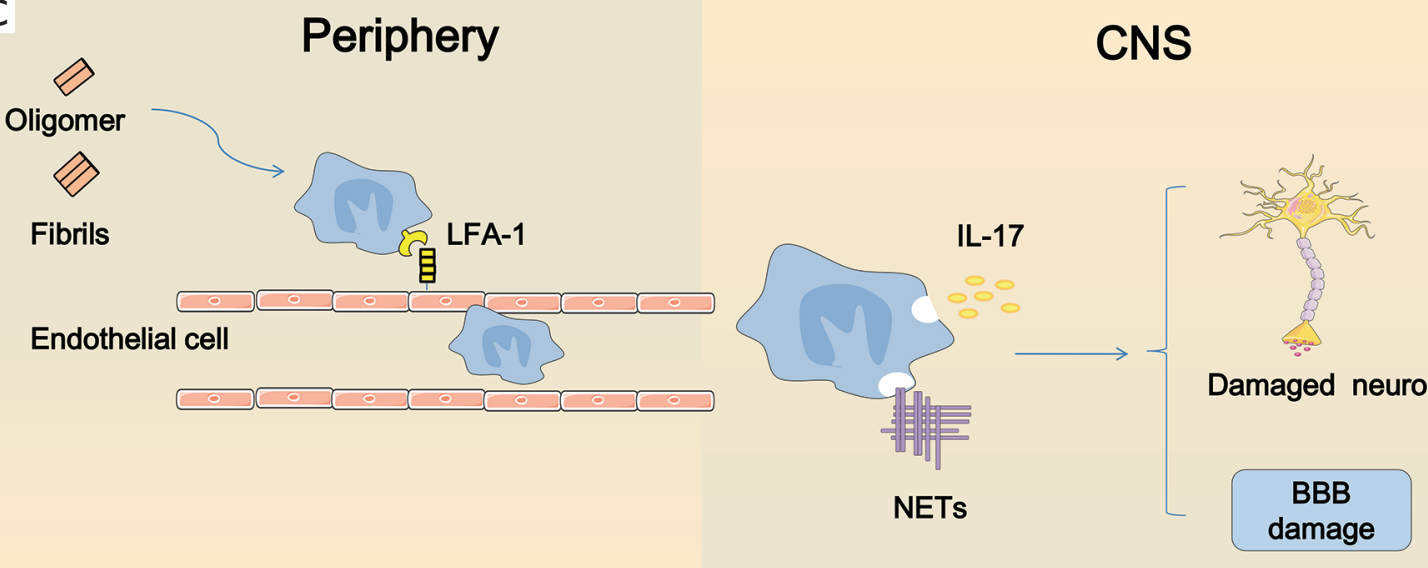

Figure 1 The process of innate immune activation in AD. (A) In the pathological fashion, APP is cleaved first by $\beta$-secretase to produce $\mathrm{sAPPb}$ and $\mathrm{C} 99$, further $\mathrm{C} 99$ is cleaved by $\gamma$-secretase to produce a more aggregation-prone and neurotoxic A $\beta$ peptide. The pathogenic $\mathrm{A} \beta$ monomers then aggregate and form A $\beta$ oligomers and fibrils; (B) extracellular A $\beta$ deposition and DAMPs as a potent stimulator binding to the PRRs on the surface of microglia trigger innate immune activation, thus result in the production and release of substantial inflammatory cytokines and other small neurotoxic molecule, such as NO and ROS, ultimately induce A $\beta$ deposition, tau phosphorylation, synapse dysfunction and neuronal loss. In addition, A $\beta$ monomers and oligomers can trigger microglial phagocytosis, subsequently degraded by IDE and NEP. Notably, the efficiency of degradation of oligomeric A $\beta$ is less than monomeric A $\beta$; (C) soluble oligomeric A $\beta$ and fibrillar A $\beta$ trigger the rapid adhesion of neutrophils via LFA-1. Subsequently neutrophils migrate into brain via LFA-1-dependent adhesion and produce neutrophils extracellular traps and IL-17. Then these productions may damage the brain blood barrier and neurons, thus contribute to the pathology of AD. BBB, blood brain barrier; AD, Alzheimer's disease; DAMPs, danger-associated molecular patterns; NO, nitric oxide; ROS, reactive oxygen species; $A \beta$, amyloid- $\beta$; IDE, insulin-degrading enzyme; $C N S$, central nervous system. 
in $\mathrm{AD}$, including inflammatory signaling transduction pathways, neuroinflammation-associated genes and the effects of inflammatory mediators on $\mathrm{AD}$ pathology.

\section{Innate immunity of central nervous system (CNS)}

\section{Function of innate immunity}

For a long time, the CNS was mistakenly considered to possess immune privilege. Indeed, brain has peculiar immune system that is different from peripheral tissues. Microglial cells are the major immune cells of cerebral innate immune system. They are highly concentrated in the gray matter-hippocampus, basal ganglia, substantia nigra, and olfactory cortex (3). The homeostasis of central nervous system primarily depends on the physiological function of microglial cells.

In the surface of microglial membrane, there are many receptors mediating signaling, such as neurotransmitter receptors, receptors for neurohormones and neuromodulators, cytokine and chemokine receptors, and pattern-recognition receptors (4). Microglia, even in a resting state, continuously scans their prescribed brain region by highly motile process to survey the existence of pathogens and cellular debris (5). In the meantime, microglia secretes brainderived neurotrophic factor (BDNF) which can promote learning-dependent synapse formation to contribute to synaptic protecting and plasticity as well as the formation of neuronal circuits (6). In addition, in the adult brain, microglia is able to influence the neurogenesis (3). In general, microglia plays a crucial part in sustaining the cerebral homeostasis, neuronal integrity, and synaptic protecting and plasticity. The depletion of microglia may lead to dysregulation of cerebral homeostasis, neuronal cell death, and synaptic dysfunction.

In addition to the parenchymal microglia, other cell types including perivascular, meningeal, and choroid plexus macrophages also play a part in the homeostasis regulation of CNS. Once the blood brain barrier (BBB) broke down on account of injury or neurodegenerative disease, bloodderived monocytes can enter the affected brain and contribute to the immune response (7).

\section{Activation of innate immunity}

In the surface of microglia, there are many PRRs containing scavenger receptors and toll-like receptors (TLRs). Danger-associated molecular patterns (DAMP) including misfolded protein, mislocalized nucleic acids and peptides, and pathogen-associated molecular patterns (PAMP) can bind to PRRs and subsequently initiate a series of immune responses, ultimately inducing the production of proinflammation cytokines by microglia.

It is well acknowledged that microglia has two phenotypes including proinflammatory (M1) form and antiinflammatory (M2) form. The classic M1 phenotype is characterized by the delivery of proinflammatory cytokines including tumor necrosis factor alpha (TNF- $\alpha$ ), interleukin (IL) $-1,-6,-12$ and -18 , accompanied with impaired phagocytic function while the alternative is marked by the delivery of anti-inflammatory cytokines containing IL-4, IL10 and transforming growth factor- $\beta$ (TGF- $\beta$ ), accompanied with increased phagocytic function. Chronic exposure to inflammation induces the conversion of microglial phenotype from proinflammatory form to antiinflammatory form. PRRs in membrane surface of microglia contribute to recognition of pathogen and DAMP (mainly $\mathrm{A} \beta$ in $\mathrm{AD}$ ), as well as endogenous molecule, such as ATP, chromogranin A, and double-stranded DNA leaked from damage neurons. $A \beta$ originates from amyloid precursor protein (APP) by means of sequential cleavage through $\beta$ and $\gamma$-secretase which is comprised of presenilin 1 (PS1). $\mathrm{A} \beta$ presents in various forms such as monomers, oligomers, protofibrils, fibrils and $A \beta$ plaques (8). Among them, $A \beta$ oligomers show the most powerful toxicity when directly added to neuronal cultures (9). Microglial cells are attracted to the injured site by $A \beta$ deposition followed by the sense of $\mathrm{A} \beta$ aggregation via TLRs. The sense subsequently initiates a series of intracellular mechanisms. Then a multi-protein signaling platform namely NLRP3 inflammasome which can control the activation of caspase- 1 and -8 is assembled. This sequential activity induces overmuch production and release of interleukin- $1 \beta$ family containing IL-18 and $-1 \beta$. However, these proinflammatory cytokines primarily appear in inactive forms that need to be activated via the cleavage through caspase- 1 or -8 , ultimately contributing to $\mathrm{AD}$ pathological mechanisms in activated forms $(10,11)$. Many lines of evidence demonstrated that NLRP3 contributes to AD pathology, and inhibition of NLRP3 could largely protect from $A \beta$ deposition and memory impairment (12). Moreover, NLRP3 inflammasome deficiency prompts microglial cells to transfer to an M2 phenotype and contributes to the decrease of $A \beta$ deposition (13). Of note, monocyte-derived macrophages (MDM) also can release proinflammatory mediators and phagocytize $\mathrm{A} \beta$ peptides. More importantly, MDMs seem to be more efficient in the 
elimination of $A \beta$ plaques when they appear in paralyzed forms. The function of MDMs is still uncertain and needs to be clearly defined (14).

In addition, neutrophils also contribute to $\mathrm{AD}$ pathology. Recent studies indicated that $A \beta$ could promote LFA1dependent adhesion of neutrophils. Furthermore, LFA-1 makes contributions to neutrophil transudation into $\mathrm{CNS}$ and the intraparenchymal motility. Primarily $\mathrm{A} \beta$ induces the conversion of LFA-1 to high-affinity state and enhances LFA1-dependent adhesion of neutrophils. Soluble oligomeric A $\beta$ induces the speedy LFA1-dependent adhesion of neutrophils. Besides, fibrillar $\mathrm{A} \beta$ also can trigger the rapid adhesion, but its effectiveness is slightly weaker than the soluble form. Subsequently neutrophils migrate to brain via the adhesion depending on LFA-1 and produce IL-17 and neutrophil extracellular traps (NETs). Neutrophil depletion or inhibition of neutrophil migration through blockage of LFA-1 integrin decreases the pathology of AD and improves memory loss (15). The production of NETs by neutrophils may damage the BBB and neurons. Another production IL-17 may have direct cytotoxicity to BBB and neurons. Besides, they may recruit more neutrophils for a vicious circle (16).

\section{Innate immune activation in AD}

\section{Genes associated with innate immune activation}

\section{Triggering receptor expressed on myeloid cells 2 (TREM2)}

TREM2 gene which encodes TREM2 protein is located in the chromosome 6p21.1 $(17,18)$. Accumulating evidence demonstrated that a functional variant $\mathrm{R} 47 \mathrm{H}$ in the TREM2 gene resulted in increased susceptibility to AD. The main cause associated with increased risk of $\mathrm{AD}$ may be the reduced function of TREM2 that resulted from $\mathrm{R} 47 \mathrm{H}$ (18-20). TREM2 proteins were mainly distributed in white matter, hippocampus and neocortex and these situations were consistent with pathological features of AD. TREM2 which signals via binding to DAP 12 (also known as TYROBP) is present in microglial cells, osteoclasts, dendritic cells and macrophages $(18,21)$. In the progress of $\mathrm{AD}$, many inflammatory cytokines are produced and released by microglia, thus leading to nearby neuron damage. Many lines of studies proved that TREM2 inhibited the production and secretion of inflammatory cytokines mediated by microglial cells, thus suppressing the inflammatory response. In addition, TREM2 also takes part in the regulation of phagocytic process, and contributes to the removal of neuronal debris as well as the possibility of $\mathrm{A} \beta$ deposition (10). The existence of $\mathrm{R} 47 \mathrm{H}$ variant may result in the reduced function of TREM2, thus leading to systematic inflammation and neuronal death. Regarding that TREM2 is expressed on microglia, the loss function of TREM2 may reduce the ability of microglia to engulf $A \beta$, thus involving the pathology of $\mathrm{AD}(18,22,23)$. In agreement with this argument, microglia with TREM2 deficiency showed appreciably diminished uptake of antibodybound $\mathrm{A} \beta$, resulting in diminished clearance of amyloid plaques (24). Furthermore, knocking down of TREM2 or DAP 12 induced decreased phagocytosis of neuronal debris and apoptotic cells. By contrast, overexpression of TREM2 enhanced the phagocytosis (22). It is another way of saying that the role of TREM2 in AD pathogenesis is primarily involving tau dysfunction (25). In short, TREM2 is present as a protective gene in a normal state for the function of inhibiting inflammatory response and regulating the phagocytosis process, whereas the existence of $\mathrm{R} 47 \mathrm{H}$ makes TREM2 to turn into a risk gene in $\mathrm{AD}$.

Interestingly, new research provided evidence to elaborate that both deficiency and hyperactivity of TREM2 can increase the risk of AD. Being different from R $47 \mathrm{H}$ which can reduce TREM2 signaling, two rare variantsD87N and T96K seemed to have the opposite effects. Colonna reported that $\mathrm{D} 87 \mathrm{~N}$ and $\mathrm{T} 96 \mathrm{~K}$ can enhance TREM2 activation. From the above, it can be seen that just promoting TREM2 activity may not lead to a beneficial result and TREM2-targeted therapies should be carefully given to achieve the optimal response (26).

\section{The transmembrane glycoprotein cluster of differentiation 33 (CD33)}

$C D 33$ which belongs to the sialic-acid-binding immunoglobulin-like lectins (SIGLECs) expressed by mature monocytes, macrophages and myeloid progenitor cells. CD33 is thought to play important roles in mediating cell-cell interactions, modulating cytokine secretion, mediating endocytosis and possibly A $\beta$ clearance (27).

Recently several independent genome-wide association studies (GWAS) indicated that CD33 was strongly associated with $\mathrm{AD}$ risk $(28,29)$. Two main single-nucleotide polymorphisms (SNPs)—rs3865444 and rs3826656, were suggested to be related to late onset Alzheimer's disease (30). Regarding rs3865444 SNP, the allele rs3865444A was thought to be relevant to reduced AD risk. Conversely, the allele rs $3865444 \mathrm{C}$ was proved to be relevant 
to increased $\mathrm{AD}$ risk $(31,32)$. In brain of $\mathrm{AD}$ patients, the levels of $C D 33$ protein and mRNA are remarkably increased. It is conceivable that up-regulation of $C D 33$ transcription in microglial cells suggests a role of $C D 33$ in the pathogenesis of $\mathrm{AD}$ (33). By contrast, rs3826656 was suggested to be related to decreased risk of AD by Yuan's research in Chinese Han population (30,34).

Mounting evidence indicated that $C D 33$ showed significant correlation with $\mathrm{A} \beta$ clearance in $\mathrm{AD}$. The $\mathrm{AD}$ protective allele rs3865444A was connected with the increase of $C D 33$ isoform lacking exon 2 which codes the $\mathrm{IgV}$ domain. This domain mediated the binding between SIGLECS family members and sialic acid, thus leading to the inhibition of phagocytosis $(32,35)$. In the brain cortex of $\mathrm{AD}$ patients carrying the rs3865444A allele, the amyloid plaque burdens were significant reduced. Conversely, the rs3865444C caused great burdens of amyloid plaque. In addition, Griciuc et al. elaborated that $C D 33$ directly modulated $\mathrm{A} \beta$ uptake by microglial cells $(30,31,33)$. Although many lines of studies have demonstrated that CD33 played complex roles in AD pathology, recent analysis of three large-scale GWAS investigating the association of 13 variants with childhood intelligence (36), cognitive performance (37), and educational attainment (38), respectively, did not show any significant association (39). This result is just the opposite of the study by Schwarz et al. which reported the protective role of $C D 33$ and other genes against postreproductive cognitive decline (40). From the above, it can be seen that the role of $C D 33$ in $\mathrm{AD}$ pathology was intricate and more problems still needed further study.

\section{Innate immune activation in $A \beta$}

\section{TLRs mediate phagocytic clearance of $A \beta$}

TLR family which expressed on the membrane surface of microglial cells are the important members of PRR. They can recognize PAMPs (invading pathogens, cell stress) and DAMPs (cellular components from damage cells) and activate the signaling molecules, including mitogenactivated protein kinase (MAPK) and transcription factor nuclear factor $\kappa \mathrm{B}(\mathrm{NF}-\kappa \mathrm{B})$, thus leading to the production and release of cytokines. Obviously, they are indispensable for maintaining the homoeostasis of CNS (41).

In the progress of $\mathrm{AD}$, three members of the TLRs family were proved to be implicated in the pathogenesis of AD, containing TLR2, TLR4 and TLR9. Among them, TLR2 and TLR4 play double-edged roles, while TLR9 only exerts a beneficial effect (Figure 2). TLR2, which is activated by axonal injury, Lewy bodies and $\mathrm{A} \beta$, gives rise to $M 1$ microglial activation, thus resulting in secretion of proinflammatory cytokines and neuronal damage (42). Moreover, TLR2 deficiency promotes microglial conversion from $M 1$ to $M 2$ state and $A \beta$ clearance, and protects neuron and synapse from $\mathrm{A} \beta$-induced neurotoxicity, thus playing a beneficial role in $\mathrm{AD}$ pathology (43). In accordance with this conception, the mice model of $\mathrm{AD}$ treated with antiTLR2 antibody showed reduced $\mathrm{A} \beta$ plaque burden, glial activation (44) and the secretion of IL- 6 and TNF- $\alpha$ from microglia (45). However, the apparently contradictory result has been reported that TLR2 activation promoted A $\beta$ uptake by microglia, thereby exerting beneficial effects on AD pathology (42). Just like TLR2, TLR4 exerts both beneficial and detrimental effects. TLR4, which is activated by $A \beta$, typical ligand-LPS, heat shock proteins (HSPs) and high-mobility group box-1 (HMGB1), involves $\mathrm{AD}$ pathogenesis by $\mathrm{A} \beta$ clearance and release of neurotoxic factors such as nitric oxide (NO). The activation of TLR4 by HMGB1 leaked from damage neurons promotes neuroinflammation, thus exacerbating AD pathology. Another effusion HSPs which originated from apoptotic and necrotic cells, especially HSP32, HSP60, HSP70, HSP90, also activate TLR4 and lead to the production of pro-inflammatory cytokines, even the CNS damage (42). By contrast, TLR4-mediated microglial activation by LPS increases A $\beta$ uptake and plays a protective role in $\mathrm{AD}$ (46). In short, TLRs exerts a beneficial role by activating microglial cells and increasing $A \beta$ uptake, while they play a detrimental role by stimulating microglia to produce pro-inflammatory cytokines. Differentiating from TLR2 and TLR4, TLR9 which is induced by cytosineguanosine-containing oligodeoxynucleotides (CpGs) only plays a beneficial role in clearance of $\mathrm{A} \beta$ during $\mathrm{AD}$ progression. Among three types of $\mathrm{CpGs}$ containing classes A, B C, CpGs-B is a strong stimulator for microglial activation. Increasing evidence demonstrated that TLR9 activation enhanced microglial phagocytosis and reduced $A \beta$ burden in brain (47). In addition, TLR 9 activation by CpGs-B was suggested to reduce the level of NFTs (48).

\section{IDE and neprilysin}

Except the microglia-mediated phagocytosis to decrease A $\beta$ level, two major endopeptidases including insulin-degrading enzyme (IDE) and neprilysin (NEP) contribute to the degradation of cytoplasmic monomeric $A \beta(49)$.

As early as 2001, Selkoe has suggested a role of IDE in $\mathrm{A} \beta$ degradation (50). Subsequently, Vepsalainen et al. 


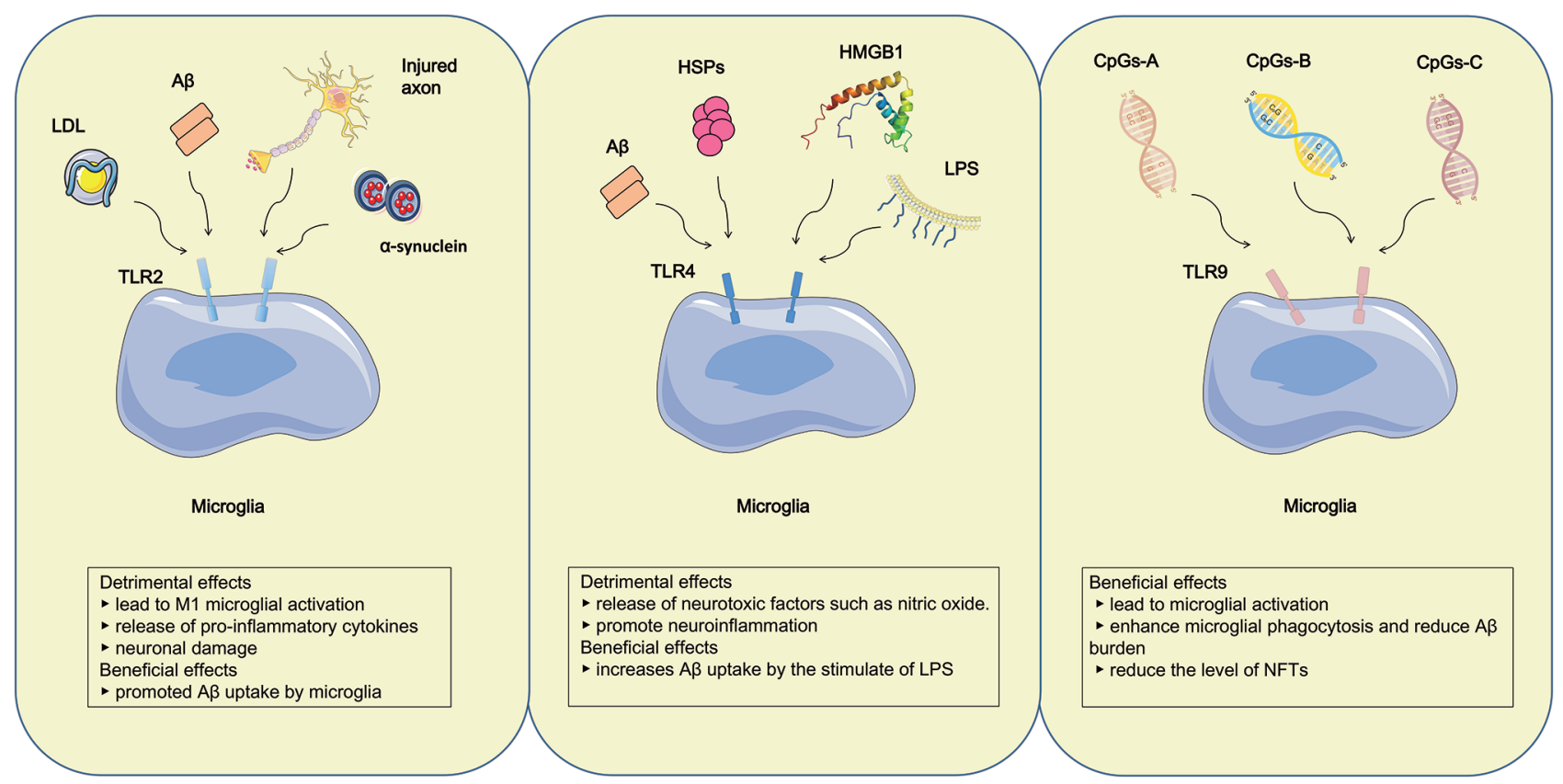

Figure 2 The effects of TLRsin AD. TLR2 which is activated by axonal injury, Lewy bodies and A $\beta$, gives rise to M1 microglial activation, thus leads to the release of proinflammatory cytokines and neuronal damage. In the contrary, TLR2 activation may also promoted A $\beta$ uptake by microglia, thereby exerts beneficial effects on AD pathology. TLR4 which is activated by A $\beta$, typical ligand-LPS, HMGB1 and HSPs, involves $\mathrm{AD}$ pathogenesis by $\mathrm{A} \beta$ clearance and release of neurotoxic factors such as nitric oxide. The activation of TLR4 by HMGB1 leaked from damage neurons promotes neuroinflammation, thus exacerbates AD pathology. Another effusion HSPs originate from apoptotic and necrotic cells, especially HSP32, HSP60, HSP70, HSP90, also activate TLR4 and lead to the production of proinflammatory cytokines, even the CNS damage. By contrast, TLR4-mediated microglial activation by LPS increases A $\beta$ uptake and plays a protective role in AD. TLR9, activated by $\mathrm{CpG}$ only plays a beneficial role in clearance of $\mathrm{A} \beta$ during $\mathrm{AD}$ progression through enhancing microglial phagocytosis and reducing A $\beta$ burden in brain. In addition, TLR9 activation by CpGs-B was suggested to reduce the level of NFTs. AD, Alzheimer's disease; HMGB1, high-mobility group box-1; HSPs, heat shock proteins; CpGs, cytosine-guanosine-containing oligodeoxynucleotides; TLRs, tolllike receptors; MAPK, mitogen-activated protein kinase; IL, interleukin.

demonstrated that IDE is genetically associated with $\mathrm{AD}$ in the Finnish population (51). In $\mathrm{AD}$, the secretion of IDE from astrocytes is mediated via an autophagy-based unconventional secretory pathway (52). Increasing evidence demonstrated that the capability of A $\beta$ degradation by IDE showed dramatic decrease that was correlated with the level of IDE protein in the early stage of AD. This suggested that decreased level of IDE can participate in the AD pathogenesis. Notably, degradation efficiency of oligomeric $\mathrm{A} \beta$ is less than that of monomeric $\mathrm{A} \beta$. As a consequence, this decrease of IDE activity leads to less $A \beta$ to be degraded (53). In addition, CALHM, as a cell-surface $\mathrm{Ca}^{2+}$ channel, has been suggested to play a role in the reduction of $A \beta$ by enhancing IDE extracellular secretion (54).

Neprilysin was suggested to be a rate-limiting enzyme implicated in the clearance of A $\beta$. Previously Leissring et al. have shown that transgenic overexpression of NEP in neurons dramatically reduced $A \beta$ levels in the brain and prevented from amyloid plaque formation (55). Subsequently, the finding was identified by a contrary genetics research that interruption of the NEP gene resulted in elevated levels of endogenous $\mathrm{A} \beta$. In addition, evidence demonstrated that NEP in cerebral cortex and hippocampus was down-regulated during the early stage of $\mathrm{AD}$ and normal aging, implying intimate relationship between NEP and AD etiology. More importantly, neuronal NEP overexpression leads to increased degradation of $\mathrm{A} \beta$, decreased formation of $\mathrm{A} \beta$ plaques and attenuation of $A \beta$-related spatial memory deficit (56). In line with this, stereotaxic injection of NEP-encoding viral vectors into hippocampus exhibited reduced $\mathrm{A} \beta$ in $\mathrm{AD}$-model mice (57). In addition, simvastatin and atorvastatin 
have been demonstrated to facilitate $A \beta$ degradation by increasing NEP secretion from astrocytes (58). Of note, monohydroxylated demethoxy-curcumin, dihydroxylation curcumin, and mono- and di-hydroxylated bisdemethoxycurcumin can increase NEP activity by amplification of mRNA and protein expression (59). These polyhydroxy curcuminoids maybe open up new horizons for the prevention of $\mathrm{AD}$.

\section{Pro-inflammatory cytokines in A $\beta$}

During the progression of $\mathrm{AD}$, microglial activation results in the production and release of a greater abundance of proinflammatory cytokines including IL-1 $\beta$, IL-6, IL18 and TNF- $\alpha$. Increasing evidence has demonstrated that the up-regulation of these pro-inflammatory cytokines play complex roles both in neuroprotection and neurodegeneration.

IL- $1 \beta$ released by microglial cells is suggested to protect from $\mathrm{AD}$. IL- $1 \beta$ can not only result in decreased production of $\mathrm{A} \beta$ by means of enhancing $\alpha$-cleavage of APP, but also mitigate amyloid pathology via increasing clearance mechanisms. With a physiological approach, APP is split via $\alpha$-secretase firstly and then via $\gamma$-secretase to generate nonpathogenic $\mathrm{p} 3$ peptide. With a pathological approach, APP is split via BACE1 to produce $\mathrm{sAPPb}$ and $\mathrm{C} 99$, and $\mathrm{C} 99$ is further split through $\gamma$-secretase to produce pathogenic $\mathrm{A} \beta$ peptide $(8,60)$. A-disintegrin-and-metalloprotease (ADAM) family proteases including ADAM-9, ADAM10 and TACE/ADAM-17, have exhibited $\alpha$-secretase activity. Emerging evidence demonstrated that IL- $1 \beta$ significantly up-regulated TACE to enhance $\alpha$-cleavage and reduce $\beta$ cleavage and $A \beta$ production. Therefore, $\alpha$-cleavage was speculated to have a neuroprotective effect in decreasing production of $A \beta$ (60). In addition, many lines of evidence proved that the overexpression of IL$1 \beta$ enhanced microglial phagocytosis and led to plaque clearance in spite of age or duration of cytokine expression (Figure 3) (61). In contrast, other researches demonstrated that IL- $1 \beta$ overexpression can impair microglial A $\beta$ clearance function (62) and induce blood-brain barrier disruption, thus increasing $A \beta$ deposition (63). From the above, it can be seen that IL- $1 \beta$ plays complex roles in $\mathrm{AD}$ and more future work in this field still needs to be done.

$\mathrm{TNF}-\alpha$, as a multifunctional pro-inflammatory cytokine, makes response to intrinsic or extrinsic stimulation. Many lines of evidence have demonstrated that TNF- $\alpha$ was associated with the onset of $\mathrm{AD}$ pathology $(64,65)$ and the level of TNF- $\alpha$ has been suggested to significantly rise in postmortem brain and cerebrospinal fluid from $\mathrm{AD}$-afflicted patients (66). Additionally, Janelsins et al. demonstrated that the level of mRNA transcripts coding TNF- $\alpha$ had a dramatically increase before the occurrence of obvious amyloid pathology in $3 \times \mathrm{Tg}-\mathrm{AD}$ mice brain (67). TNF- $\alpha$ was produced in excess close to the amyloid plaques and seemed to reflect the pathologic progression of AD (62). TNF- $\alpha$ has two cognate transmembrane receptors-containing TNF-RI and -RII, which are differentially expressed and regulated. Knocking out TNF-RI in the brains of $3 \times \mathrm{Tg}$ $\mathrm{AD}$ mice can prevent $\mathrm{AD}$-related amyloid pathology (68) while long-standing global inhibition of TNF-R signaling in 3xTg-AD mice through TNF-RI and TNF-RII ablation in all cell types results in an enhanced increase of amyloid and NFT pathology (69). Furthermore, genetic ablation of TNF-RII gene strengthens AD-like pathology in APP mice (70). It's likely to indicate that TNF-RII which exerts protective effects may neutralize the detrimental role of TNF-RI. Consistent with this, suppression of soluble TNF signaling precluded pre-plaque amyloid-associated neuropathology in an AD mouse model. This conclusion suggests that soluble TNF acts as crucial mediators in early pathology in $3 \times \mathrm{TgAD}$ mice (68). TNF- $\alpha$ is thought to be a pleiotropic pro-inflammatory cytokine and its intricate relationship with $\mathrm{AD}$ needs more research.

Except IL- $1 \beta$ and TNF- $\alpha$, other proinflammatory cytokines such as IL-6, IL-12/IL-23, and IL-18, also participate in AD (Table 1). Increased level of IL-12p40 (a subunit shared by IL-23 and IL-12) was found in cerebrospinal fluid of patients with $\mathrm{AD}$, suggesting that IL12/IL-23 signaling pathways were triggered. In accordance with this assumption, genetic knockout of the IL-12/IL23 signaling molecules $\mathrm{p} 19, \mathrm{p} 35$ or p40 led to reduced amyloid load in brain. In addition, intracerebroventricular administration of $\mathrm{p} 40$-specific neutralizing antibodies reduced soluble $A \beta$ peptides levels and enhanced spatial memory. It is notable that IL-23 and IL-12 are mainly produced by microglial cells. Due to high expression of the IL-23 receptors in the astrocytes, microglial p40 may stimulate the uptake of amyloid peptides by astrocytes (71).

\section{Anti-inflammatory cytokine IL-10 in A $\beta$}

In brains of $\mathrm{AD}$ patients, elevated level of anti-inflammatory cytokine IL-10 played a role in AD. IL-10 signaling is triggered via combination of IL-10 homodimer and its cognate receptor (IL-10R), and it in turn results in 


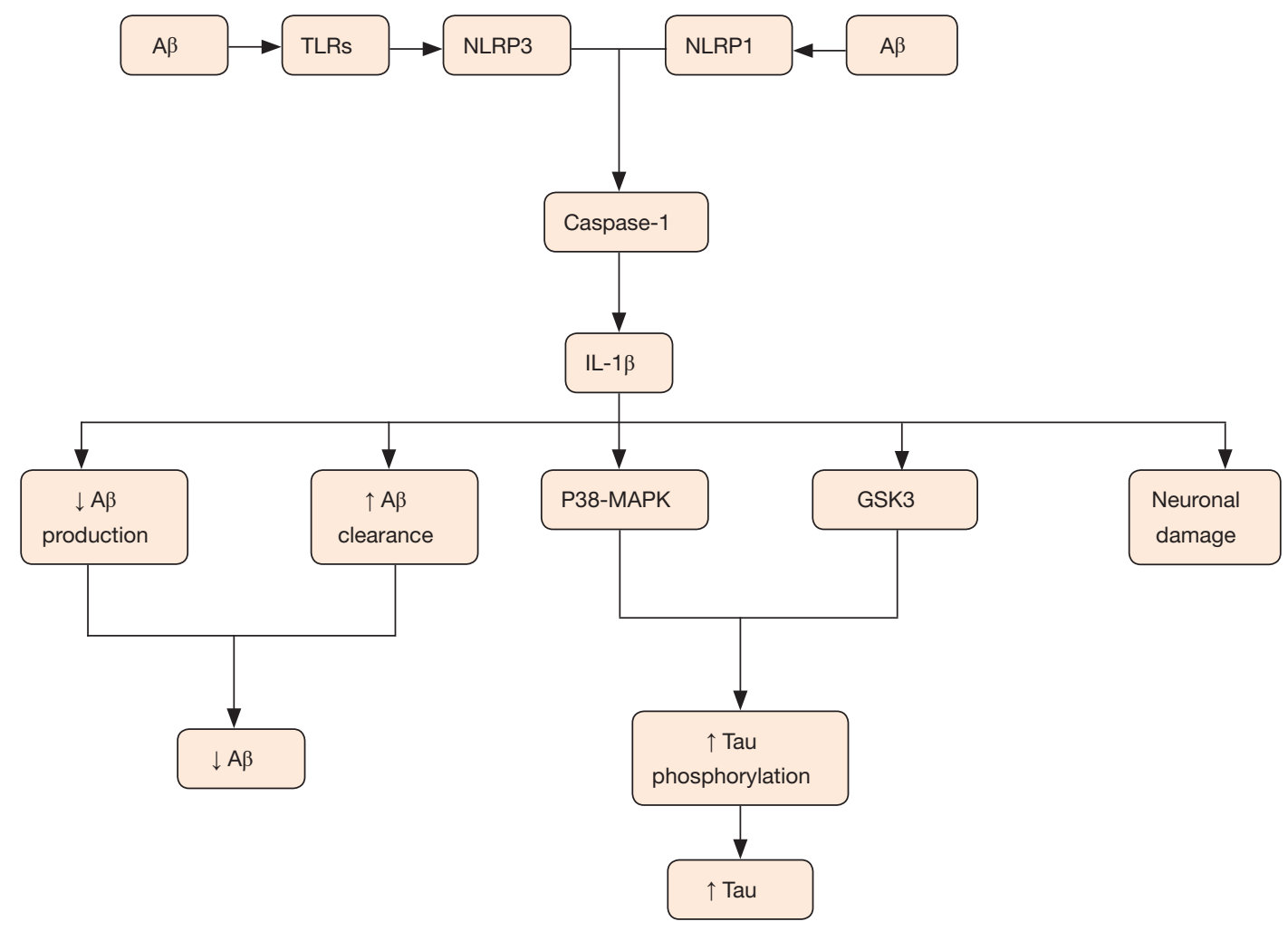

Figure 3 The Production and effects of IL-1ßin AD. The recognition of A $\beta$ by TLRs initiates a series of intracellular mechanism. Then a multi-protein signaling platform namely NLRP3 and NLRP1 inflammasome is assembled which can control the activation of caspase-1 and caspase-8. This sequential activity induces the overmuch production and release of interleukin-1 $\beta$ family containing IL-18 and IL-1 $\beta$. IL$1 \beta$ which is released by microglial cells plays complex roles in AD pathology. IL-1 $\beta$ plays a neuroprotective role by reduced production and increased clearance of A $\beta$. Conversely, elevated levels of IL-1 $\beta$ aggravate tau protein phosphorylation and tangle formation via the activation of abnormal p38-MAPK and GSK3, thus aggravating tau pathology. In addition, long-term exposure to overexpression of IL-1 $\beta$ causes neuronal damage. GSK3, glycogen synthase kinase3; AD, Alzheimer's disease; TLRs, toll-like receptors; IL, interleukin; A $\beta$, amyloid- $\beta$; MAPK, mitogen-activated protein kinase. $\uparrow$, increase; $\downarrow$, decrease.

Table 1 Effects of inflammatory cytokines in Alzheimer's disease pathology

\begin{tabular}{|c|c|c|c|}
\hline Inflammatory cytokines & Effect on $A \beta$ & Effect on tau & Effect on neurons \\
\hline \multicolumn{4}{|c|}{ Pro-inflammatory cytokines } \\
\hline IL-1 $\beta$ & $\begin{array}{l}\text { Reduce } A \beta \text { production; increase } A \beta \\
\text { clearance }\end{array}$ & $\begin{array}{l}\text { Increase tau phosphorylation; increase } \\
\text { tau pathology }\end{array}$ & $\begin{array}{l}\text { Increase neuronal death anc } \\
\text { damage }\end{array}$ \\
\hline IL-6 & Reduce $A \beta$ deposition & Increase tau phosphorylation & Rescue neurons \\
\hline IL-12/IL-23 & Reduce $A \beta$ clearance & Increase tau phosphorylation & Reduce neuronal loss \\
\hline TNF- $\alpha$ & $\begin{array}{l}\text { Increase } A \beta \text { deposition; reduce } A \beta \\
\text { clearance }\end{array}$ & Increase tau hyperphosphorylation & $\begin{array}{l}\text { Increase neuronal death anc } \\
\text { damage }\end{array}$ \\
\hline \multicolumn{4}{|c|}{ Anti-inflammatory cytokines } \\
\hline IL-10 & $\begin{array}{l}\text { Reduce } A \beta \text { clearance; increase } A \beta \\
\text { deposition }\end{array}$ & No significant change & Indirect damage and death \\
\hline
\end{tabular}

$\mathrm{A} \beta$, amyloid beta; IL, interleukin; TNF- $\alpha$, tumor necrosis factor alpha; TGF- $\beta$, transforming growth factor- $\beta$. 
phosphorylation of relevant Janus kinase 1, downstream phosphorylation, as well as activation of signal transducer and activator of transcription 3 (STAT3). Afterwards, phosphorylated STAT3 is delivered to nucleus and it dominates the transcription of inflammatory genes (72).

Increasing evidence demonstrated that anti-inflammatory cytokine IL-10 was involved in AD pathogenesis. Chakrabarty et al. identified that IL-10 increased A $\beta$ accumulation and exacerbated memory impairment in APP mouse brains (73). In line with this result, GuillotSestier et al. also proved that IL-10 signaling inhibited A $\beta$ clearance by microglia and increased the concentration of cerebral A $\beta$. In addition, IL-10 deficiency alleviated A $\beta$ deposition and mitigated synaptic toxicity and cognitive deficit in APP/PS1 mice. The rescue of synaptic toxicity and memory impairment may be owed to the rebalance of innate immune system (72). Furthermore, $A \beta$ deposition presents as a more diffuse morphology and microglial cells are inclined to penetrate and fragment the core of plaques in IL-10 mice (74). A novel perspective has been a focus that overexpression of IL-10 increased the transcription of ApoE and inhibited the $A \beta$ degradation by microglia. The mechanism is likely to be that apolipoproteins which can highly bind to $\mathrm{A} \beta$ hinder microglial phagocytosis $(73,74)$.

\section{NO and iNOS in A}

Some small molecules such as NO and inducible nitric oxide synthase (iNOS) also have an exhaustive role in inflammatory mechanism during AD progression. Increasing evidence demonstrated that oxidative and nitrosative damage may be crucial in the pathogenesis of $\mathrm{AD}$ (75). To react to proinflammatory cytokines, glial cells and neurons generate iNOS in AD. Subsequently, elevated expression level of iNOS can result in enhanced generation of local NO which is speculated to be implicated in inhibition of mitochondrial respiration, axonal and synaptic damage. In addition, iNOS can also enhance neuronal apoptosis that induces neurodegenerative process $(10,11)$. A high concentration of iNOS-derived NO results in the production of peroxynitrite which can lead to nitration of tyrosine residue at position 10 of $\mathrm{A} \beta$ peptide. During $\mathrm{AD}$ process, iNOS-mediated nitration of $A \beta$ has an increased propensity to aggregate and to form the core of $A \beta$ plaques (76). Moreover, the nitrated $\mathrm{A} \beta$ can suppress synaptic plastic more powerful and shortage of iNOS significantly prevented AD-like mice from untimely death, formation of plaque in brain, boosted $A \beta$ levels, microgliosis, astrocytosis and protein tyrosine nitration (77).

\section{Innate immune activation in tau pathology}

\section{IL-1 $\beta$ in tau pathology}

One of AD pathological hallmarks is intra-neuronal NFTs formed after $A \beta$ deposition which occurs in early stage of AD. NFTs comprise of hyperphosphorylated tau proteins which normally stabilize neuronal cytoskeleton microtubule proteins. Hyperphosphorylation expels tau from the surface of microtubule, which may lead to the accumulation of toxic tau peptides, compromised axonal integrity, as well as the formation of filaments and tangles from tau (78). The formation of NFTs subsequently leads to diverse cellular changes which result in neuron degeneration (10). Microglia-driven neuroinflammation is known to promote tau phosphorylation and aggregation and is thought to be one cause of the formation of NFTs. Sheng et al. have demonstrated that chronic excessive expression of IL-1 remarkably raised relevant tau mRNA level and accelerated the phosphorylation of tau proteins, thus suggesting a role of IL-1 in characteristic neurofibrillary changes of $\mathrm{AD}$ pathogenesis (79). After that, a growing number of evidence has proven the finding that elevated levels of IL$1 \beta$ aggravated tau protein phosphorylation and tangle formation via the activation of abnormal p38-MAPK and glycogen synthase kinase 3 (GSK3) $(80,81)$. Furthermore, blocking IL-1 signaling decreased the activity of several tau kinases, including p38-MAPK, GSK-3b, and cdk5/p25, as well as declined levels of phosphorylated tau. Therefore, inhibition of IL-1 signaling significantly mitigated tau pathology, as well as alleviated cognitive deficits (82). All in all, IL-1 $\beta$ can induce the neurodegenerative events through increasing tau level and aggravating tau pathology (Figure 3).

\section{CX3CR1 in tau pathology}

CX3CR1 is a deta chemokine receptor expressed in microglial cells in the brain. Fractalkine contains two isoforms including soluble isoform and anchoredmembrane isoform. Among them, soluble isoform maintains microglial cells in a suitable state by means of interaction with CX3CR1 (83). The deficiency of CX3CR1 caused the acceleration of tau pathology and memory deficits in an htau mouse model of tauopathy (84). As Bhaskar et al. demonstrated, CX3CR1 deficiency can increase microglial activation and enhance tau pathology as well as cause behavioral abnormalities (85). However, CX3CR1 deficiency resulted in altered microglial activation and gene dose-dependent $\mathrm{A} \beta$ elimination (83). As known to all, the anchored membrane fractalkine signaling might 
increase amyloid pathology. Conversely, soluble fractalkine levels could preclude tau pathology. The deficiency of the anchored membrane fractalkine accelerated tau phosphorylation and reduced amyloid deposition in opposite (83). It is notable that deletion of the CX3CR1 gene is suggested to be protective against neuronal loss in the triple transgenic mouse model of $\mathrm{AD}$ (86), while it will induce neurotoxicity in mouse models of systemic inflammation, PD, and ALS (87). As aforementioned, CX3CR1 plays a different role in amyloid beta clearance and tau pathology. The deficiency of CX3CR1 induces microglial phagocytosis, reduces amyloid burden, and accelerates tau phosphorylation and aggregation.

\section{Further direction in clinic}

\section{Inflammatory biomarkers in CSF for early diagnosis}

Although significant progression has been made in our understanding of $\mathrm{AD}$ pathogenesis, there is few effective measures for preventing or delaying the occurrence of this disease. Researchers have shifted their focus to the diagnosis and treatment in the early stage of $\mathrm{AD}$ as well as the stage before the occurrence of cognitive impairment. Three classical biomarkers comprised of total tau, phosphorylated tau and amyloid- $\beta$ have been identified (88). Since innate immune activation involves in the development of AD pathology, whether inflammatory mediators can be biomarkers for early diagnosis should be investigated.

By far, several studies have investigated the levels of proinflammatory and anti-inflammatory cytokines in CSF of MCI and AD patients. However, these studies have shown controversial results. These contradictory results are attributable to the patient characterization and detection methods, and the most important cause may be the disease progression which is in correlation with cytokine expression (89). In a comparative overview about the levels of body fluid cytokines in MCI and AD patients, researchers suggested that cytokines like TNF- $\alpha$, IL-6 or IL- $1 \beta$ might rise slowly while MCP-1, IL-18 or IP-10 might come to a peak in the stage of conversion from MCI to AD. Nevertheless, these slowly increased cytokines are so difficult to detect that they are not recommended as suitable biomarkers for AD. Fortunately, the cytokines that show a peak may be priming biomarkers (90). Of note, the levels of cytokines in CSF assist with the diagnosis of $\mathrm{AD}$ on the basis of the existing classical biomarkers. Present studies are short of longitudinal data over years of cytokine expression that may cause the contradictory results. Standardization of experimental protocol and long standing longitudinal sampling are urgently needed to conquer the interindividual discrepancies.

\section{Inflammation as a potential therapeutic target for Alzheimer's disease}

Epidemiologic evidence has suggested an affirmative influence of non-steroidal anti-inflammatory drugs (NSAIDs) in AD. However, several randomised trials with NSAIDs seemed to be not exactly successful (91-93), while some early trials demonstrated reduced cognitive impairment in $\mathrm{AD}$ patients with NSAIDs treatment (94). Furthermore, an adverse effect has been showed on AD pathology in the late stage with naproxen or celecoxib treatment despite the positive effect in the early stage (95). Thereby, the effects of NSAIDs on AD pathology may depend on the time point-namely the stage of disease, and the choice of specific NSAIDs.

During AD development, microglial cells exposing to DAMP or PAMP transform from a beneficial form to a detrimental form via proinflammatory signaling transduction such as NLRP3 inflammasome. Blocking the signaling pathway as a new therapeutic method for $\mathrm{AD}$ may hold promise. To this end, the nuclear hormone receptor PPAR- $\gamma$ was regarded as a therapeutic target for pharmacologic agonist such as the thiazolidinedione class of anti-diabetics (96). These agonists of PPAR- $\gamma$ can potently stimulate $A \beta$ uptake by microglia through the up-regulation of CD36 expression (97). It is notable that the specificity of agonists which means to be exclusively restricted to microglia and not having crucial functions in other cell types is necessary for successful modification of these pathways (98). In addition, several NSAIDs which are demonstrated to protect from $\mathrm{AD}$ are powerful activators of PPAR- $\gamma$, and may contribute to molecular mechanisms (11).

In general, pharmacologic intervention in different disease stages shows disparate effects. Therefore, the optimal timing of intervention seems to have great significance to the clinical efficacy of therapeutic methods. In the future, more and more work needs to be done in this field of delaying and preventing the progression of $\mathrm{AD}$.

\section{Conclusions and future perspectives}

Up to now, the mechanism underlying innate immune activation has shown a little bit clearness. However, many 
problems still need to be solved. To seek therapeutic strategies for $\mathrm{AD}$ to delay or prevent the progression of this disease, more and more studies need to be conducted. The failure of large clinical trials raises the doubt whether NSAIDs can benefit all AD patients by anti-inflammatory interventions. In addition, the time point, duration, and the specificity of NSAIDs to treat $\mathrm{AD}$ need more investigation. The early diagnosis of $\mathrm{AD}$ exerts significant effects on the disease precaution and prognosis. With the development of modern technology, more and more biomarkers were found in CSF of AD patients. However, these detection methods don't seem to be accessible and valid. Therefore, operability and accurate biomarkers are crucial to the diagnosis of AD. Moreover, blocking the inflammation signaling pathways might be a promising disease-modifying treatment in disease course. More in-depth investigations are required in the aspect of innate immunity for therapeutic strategies.

\section{Acknowledgements}

This work was supported by grants from the Shandong Taishan Scholar, Qingdao Key Health Discipline Development Fund, and Shandong Provincial Collaborative Innovation Center for Neurodegenerative Disorders.

\section{Footnote}

Conflicts of Interest: The authors have no conflicts of interest to declare.

\section{References}

1. Scheltens P, Blennow K, Breteler MM, et al. Alzheimer's disease. Lancet 2016;388:505-17.

2. Wood H. Alzheimer disease: Meta-analysis finds high reversion rate from MCI to normal cognition. Nat Rev Neurol 2016;12:189.

3. Benarroch EE. Microglia: Multiple roles in surveillance, circuit shaping, and response to injury. Neurology 2013;81:1079-88.

4. Kettenmann H, Hanisch UK, Noda M, et al. Physiology of microglia. Physiol Rev 2011;91:461-553.

5. Nayak D, Roth TL, McGavern DB. Microglia development and function. Annu Rev Immunol 2014;32:367-402.

6. Parkhurst CN, Yang G, Ninan I, et al. Microglia promote learning-dependent synapse formation through brainderived neurotrophic factor. Cell 2013;155:1596-609.
7. Meyer-Luehmann M, Prinz M. Myeloid Cells in Alzheimer's Disease: Culprits, Victims or Innocent Bystanders? Trends Neurosci 2015;38:659-68.

8. Heppner FL, Ransohoff RM, Becher B. Immune attack: the role of inflammation in Alzheimer disease. Nat Rev Neurosci 2015;16:358-72.

9. Walsh DM, Klyubin I, Fadeeva JV, et al. Naturally secreted oligomers of amyloid beta protein potently inhibit hippocampal long-term potentiation in vivo. Nature 2002;416:535-9.

10. Heneka MT, Kummer MP, Latz E. Innate immune activation in neurodegenerative disease. Nat Rev Immunol 2014;14:463-77.

11. Heneka MT, Golenbock DT, Latz E. Innate immunity in Alzheimer's disease. Nat Immunol 2015;16:229-36.

12. Tan MS, Yu JT, Jiang T, et al. The NLRP3 inflammasome in Alzheimer's disease. Mol Neurobiol 2013;48:875-82.

13. Heneka MT, Kummer MP, Stutz A, et al. NLRP3 is activated in Alzheimer's disease and contributes to pathology in APP/PS1 mice. Nature 2013;493:674-8.

14. Martin E, Boucher C, Fontaine B, et al. Distinct inflammatory phenotypes of microglia and monocytederived macrophages in Alzheimer's disease models: effects of aging and amyloid pathology. Aging Cell 2017;16:27-38.

15. Zenaro E, Pietronigro E, Della Bianca V, et al. Neutrophils promote Alzheimer's disease-like pathology and cognitive decline via LFA-1 integrin. Nat Med 2015;21:880-6.

16. Allen C, Thornton P, Denes A, et al. Neutrophil cerebrovascular transmigration triggers rapid neurotoxicity through release of proteases associated with decondensed DNA. J Immunol 2012;189:381-92.

17. Colonna M. TREMs in the immune system and beyond. Nat Rev Immunol 2003;3:445-53.

18. Jiang T, Yu JT, Zhu XC, et al. TREM2 in Alzheimer's disease. Mol Neurobiol 2013;48:180-5.

19. Karch CM, Goate AM. Alzheimer's disease risk genes and mechanisms of disease pathogenesis. Biol Psychiatry 2015;77:43-51.

20. Chouraki V, Seshadri S. Genetics of Alzheimer's disease. Adv Genet 2014;87:245-94.

21. Jin SC, Benitez BA, Karch CM, et al. Coding variants in TREM2 increase risk for Alzheimer's disease. Hum Mol Genet 2014;23:5838-46.

22. Jiang T, Tan L, Zhu XC, et al. Upregulation of TREM2 ameliorates neuropathology and rescues spatial cognitive impairment in a transgenic mouse model of Alzheimer's disease. Neuropsychopharmacology 2014;39:2949-62. 
23. Colonna M, Wang Y. TREM2 variants: new keys to decipher Alzheimer disease pathogenesis. Nat Rev Neurosci 2016;17:201-7.

24. Xiang X, Werner G, Bohrmann B, et al. TREM2 deficiency reduces the efficacy of immunotherapeutic amyloid clearance. EMBO Mol Med 2016;8:992-1004.

25. Malkki H. Alzheimer disease: The involvement of TREM2 $\mathrm{R} 47 \mathrm{H}$ variant in Alzheimer disease confirmed, but mechanisms remain elusive. Nat Rev Neurol 2015;11:307.

26. Wood H. Alzheimer disease: TREM-2 signalling and Alzheimer disease - striking the right balance. Nat Rev Neurol 2016;12:556.

27. Crocker PR, Redelinghuys P. Siglecs as positive and negative regulators of the immune system. Biochem Soc Trans 2008;36:1467-71.

28. Tan L, Yu JT, Zhang W, et al. Association of GWAS-linked loci with late-onset Alzheimer's disease in a northern Han Chinese population. Alzheimers Dement 2013;9:546-53.

29. Kamboh MI, Demirci FY, Wang X, et al. Genome-wide association study of Alzheimer's disease. Transl Psychiatry 2012;2:e117.

30. Jiang T, Yu JT, Hu N, et al. CD33 in Alzheimer's disease. Mol Neurobiol 2014;49:529-35.

31. Bradshaw EM, Chibnik LB, Keenan BT, et al. CD33 Alzheimer's disease locus: altered monocyte function and amyloid biology. Nat Neurosci 2013;16:848-50.

32. Villegas-Llerena C, Phillips A, Garcia-Reitboeck P, et al. Microglial genes regulating neuroinflammation in the progression of Alzheimer's disease. Curr Opin Neurobiol 2016;36:74-81.

33. Griciuc A, Serrano-Pozo A, Parrado AR, et al. Alzheimer's disease risk gene CD33 inhibits microglial uptake of amyloid beta. Neuron 2013;78:631-43.

34. Yuan Q, Chu C, Jia J. Association studies of 19 candidate SNPs with sporadic Alzheimer's disease in the North Chinese Han population. Neurol Sci 2012;33:1021-8.

35. Malik M, Simpson JF, Parikh I, et al. CD33 Alzheimer's risk-altering polymorphism, CD33 expression, and exon 2 splicing. J Neurosci 2013;33:13320-5.

36. Benyamin B, Pourcain B, Davis OS, et al. Childhood intelligence is heritable, highly polygenic and associated with FNBP1L. Mol Psychiatry 2014;19:253-8.

37. Rietveld CA, Esko T, Davies G, et al. Common genetic variants associated with cognitive performance identified using the proxy-phenotype method. Proc Natl Acad Sci U S A 2014;111:13790-4.

38. Rietveld CA, Medland SE, Derringer J, et al. GWAS of 126,559 individuals identifies genetic variants associated with educational attainment. Science 2013;340:1467-71.

39. Liu G, Jiang Q. Alzheimer's disease CD33 rs3865444 variant does not contribute to cognitive performance. Proc Natl Acad Sci U S A 2016;113:E1589-90.

40. Schwarz F, Springer SA, Altheide TK, et al. Humanspecific derived alleles of CD33 and other genes protect against postreproductive cognitive decline. Proc Natl Acad Sci U S A 2016;113:74-9.

41. Drouin-Ouellet J, Cicchetti F. Inflammation and neurodegeneration: the story 'retolled'. Trends Pharmacol Sci 2012;33:542-51.

42. Su F, Bai F, Zhou H, et al. Microglial toll-like receptors and Alzheimer's disease. Brain Behav Immun 2016;52:187-98.

43. Liu S, Liu Y, Hao W, et al. TLR2 is a primary receptor for Alzheimer's amyloid beta peptide to trigger neuroinflammatory activation. J Immunol 2012;188:1098-107.

44. McDonald CL, Hennessy E, Rubio-Araiz A, et al. Inhibiting TLR2 activation attenuates amyloid accumulation and glial activation in a mouse model of Alzheimer's disease. Brain Behav Immun 2016;58:191-200.

45. Costello DA, Carney DG, Lynch MA. alpha-TLR2 antibody attenuates the Abeta-mediated inflammatory response in microglia through enhanced expression of SIGIRR. Brain Behav Immun 2015;46:70-9.

46. Tahara K, Kim HD, Jin JJ, et al. Role of toll-like receptor signalling in Abeta uptake and clearance. Brain 2006;129:3006-19.

47. Doi Y, Mizuno T, Maki Y, et al. Microglia activated with the toll-like receptor 9 ligand $\mathrm{CpG}$ attenuate oligomeric amyloid \{beta\} neurotoxicity in in vitro and in vivo models of Alzheimer's disease. Am J Pathol 2009;175:2121-32.

48. Scholtzova H, Chianchiano P, Pan J, et al. Amyloid beta and Tau Alzheimer's disease related pathology is reduced by Toll-like receptor 9 stimulation. Acta Neuropathol Commun 2014;2:101.

49. Tanzi RE, Moir RD, Wagner SL. Clearance of Alzheimer's Abeta peptide: the many roads to perdition. Neuron 2004;43:605-8.

50. Selkoe DJ. Clearing the brain's amyloid cobwebs. Neuron 2001;32:177-80.

51. Vepsalainen S, Parkinson M, Helisalmi S, et al. Insulindegrading enzyme is genetically associated with Alzheimer's disease in the Finnish population. J Med Genet 2007;44:606-8.

52. Son SM, Cha MY, Choi H, et al. Insulin-degrading enzyme secretion from astrocytes is mediated by an 
autophagy-based unconventional secretory pathway in Alzheimer disease. Autophagy 2016;12:784-800.

53. Stargardt A, Gillis J, Kamphuis W, et al. Reduced amyloidbeta degradation in early Alzheimer's disease but not in the APPswePS1dE9 and 3xTg-AD mouse models. Aging Cell 2013;12:499-507.

54. Vingtdeux V, Chandakkar P, Zhao H, et al. CALHM1 ion channel elicits amyloid-beta clearance by insulin-degrading enzyme in cell lines and in vivo in the mouse brain. J Cell Sci 2015;128:2330-8.

55. Leissring MA, Farris W, Chang AY, et al. Enhanced proteolysis of beta-amyloid in APP transgenic mice prevents plaque formation, secondary pathology, and premature death. Neuron 2003;40:1087-93.

56. Poirier R, Wolfer DP, Welzl H, et al. Neuronal neprilysin overexpression is associated with attenuation of Abeta-related spatial memory deficit. Neurobiol Dis 2006;24:475-83.

57. Iwata N, Sekiguchi M, Hattori Y, et al. Global brain delivery of neprilysin gene by intravascular administration of AAV vector in mice. Sci Rep 2013;3:1472.

58. Yamamoto N, Fujii Y, Kasahara R, et al. Simvastatin and atorvastatin facilitates amyloid beta-protein degradation in extracellular spaces by increasing neprilysin secretion from astrocytes through activation of MAPK/Erk1/2 pathways. Glia 2016;64:952-62.

59. Chen PT, Chen ZT, Hou WC, et al. Polyhydroxycurcuminoids but not curcumin upregulate neprilysin and can be applied to the prevention of Alzheimer's disease. Sci Rep 2016;6:29760.

60. Tachida Y, Nakagawa K, Saito T, et al. Interleukin-1 beta up-regulates TACE to enhance alpha-cleavage of APP in neurons: resulting decrease in Abeta production. J Neurochem 2008;104:1387-93.

61. Matousek SB, Ghosh S, Shaftel SS, et al. Chronic IL1beta-mediated neuroinflammation mitigates amyloid pathology in a mouse model of Alzheimer's disease without inducing overt neurodegeneration. J Neuroimmune Pharmacol 2012;7:156-64.

62. Wang WY, Tan MS, Yu JT, et al. Role of pro-inflammatory cytokines released from microglia in Alzheimer's disease. Ann Transl Med 2015;3:136.

63. Wang Y, Jin S, Sonobe Y, et al. Interleukin-1beta induces blood-brain barrier disruption by downregulating Sonic hedgehog in astrocytes. PLoS One 2014;9:e110024.

64. Di Bona D, Candore G, Franceschi C, et al. Systematic review by meta-analyses on the possible role of TNF-alpha polymorphisms in association with Alzheimer's disease.
Brain Res Rev 2009;61:60-8.

65. Swardfager W, Lanctot K, Rothenburg L, et al. A metaanalysis of cytokines in Alzheimer's disease. Biol Psychiatry 2010;68:930-41.

66. McGeer PL, McGeer EG. Local neuroinflammation and the progression of Alzheimer's disease. J Neurovirol 2002;8:529-38.

67. Janelsins MC, Mastrangelo MA, Park KM, et al. Chronic neuron-specific tumor necrosis factor-alpha expression enhances the local inflammatory environment ultimately leading to neuronal death in $3 \times \mathrm{Tg}-\mathrm{AD}$ mice. Am J Pathol 2008;173:1768-82.

68. McAlpine FE, Lee JK, Harms AS, et al. Inhibition of soluble TNF signaling in a mouse model of Alzheimer's disease prevents pre-plaque amyloid-associated neuropathology. Neurobiol Dis 2009;34:163-77.

69. Montgomery SL, Narrow WC, Mastrangelo MA, et al. Chronic neuron- and age-selective down-regulation of TNF receptor expression in triple-transgenic Alzheimer disease mice leads to significant modulation of amyloid- and Tau-related pathologies. Am J Pathol 2013;182:2285-97.

70. Jiang H, He P, Xie J, et al. Genetic deletion of TNFRII gene enhances the Alzheimer-like pathology in an APP transgenic mouse model via reduction of phosphorylated IkappaBalpha. Hum Mol Genet 2014;23:4906-18.

71. Vom Berg J, Prokop S, Miller KR, et al. Inhibition of IL-12/ IL-23 signaling reduces Alzheimer's disease-like pathology and cognitive decline. Nat Med 2012;18:1812-9.

72. Guillot-Sestier MV, Doty KR, Gate D, et al. Il10 deficiency rebalances innate immunity to mitigate Alzheimer-like pathology. Neuron 2015;85:534-48.

73. Chakrabarty P, Li A, Ceballos-Diaz C, et al. IL-10 alters immunoproteostasis in APP mice, increasing plaque burden and worsening cognitive behavior. Neuron 2015;85:519-33.

74. Michaud JP, Rivest S. Anti-inflammatory signaling in microglia exacerbates Alzheimer's disease-related pathology. Neuron 2015;85:450-2.

75. Butterfield DA, Reed TT, Perluigi M, et al. Elevated levels of 3-nitrotyrosine in brain from subjects with amnestic mild cognitive impairment: implications for the role of nitration in the progression of Alzheimer's disease. Brain Res 2007;1148:243-8.

76. Kummer MP, Hermes M, Delekarte A, et al. Nitration of tyrosine 10 critically enhances amyloid beta aggregation and plaque formation. Neuron 2011;71:833-44.

77. Nathan C, Calingasan N, Nezezon J, et al. Protection 
from Alzheimer's-like disease in the mouse by genetic ablation of inducible nitric oxide synthase. J Exp Med 2005;202:1163-9.

78. Drewes G. MARKing tau for tangles and toxicity. Trends Biochem Sci 2004;29:548-55.

79. Sheng JG, Zhu SG, Jones RA, et al. Interleukin-1 promotes expression and phosphorylation of neurofilament and tau proteins in vivo. Exp Neurol 2000;163:388-91.

80. Ghosh S, Wu MD, Shaftel SS, et al. Sustained interleukin1beta overexpression exacerbates tau pathology despite reduced amyloid burden in an Alzheimer's mouse model. J Neurosci 2013;33:5053-64.

81. Liu L, Chan C. The role of inflammasome in Alzheimer's disease. Ageing Res Rev 2014;15:6-15.

82. Kitazawa M, Cheng D, Tsukamoto MR, et al. Blocking IL-1 signaling rescues cognition, attenuates tau pathology, and restores neuronal beta-catenin pathway function in an Alzheimer's disease model. J Immunol 2011;187:6539-49.

83. Merino JJ, Muneton-Gomez V, Alvarez MI, et al. Effects of CX3CR1 and Fractalkine Chemokines in Amyloid Beta Clearance and p-Tau Accumulation in Alzheimer's Disease (AD) Rodent Models: Is Fractalkine a Systemic Biomarker for AD? Curr Alzheimer Res 2016;13:403-12.

84. Maphis N, Xu G, Kokiko-Cochran ON, et al. Reactive microglia drive tau pathology and contribute to the spreading of pathological tau in the brain. Brain 2015;138:1738-55.

85. Bhaskar K, Konerth M, Kokiko-Cochran ON, et al. Regulation of tau pathology by the microglial fractalkine receptor. Neuron 2010;68:19-31.

86. Fuhrmann M, Bittner T, Jung CK, et al. Microglial $\mathrm{Cx} 3 \mathrm{cr} 1$ knockout prevents neuron loss in a mouse model of Alzheimer's disease. Nat Neurosci 2010;13:411-3.

87. Cardona AE, Pioro EP, Sasse ME, et al. Control of microglial neurotoxicity by the fractalkine receptor. Nat Neurosci 2006;9:917-24.

88. Blennow K, Hampel H, Weiner M, et al. Cerebrospinal

Cite this article as: Wang MM, Miao D, Cao XP, Tan L, Tan L. Innate immune activation in Alzheimer's disease. Ann Transl Med 2018;6(10):177. doi: 10.21037/atm.2018.04.20 fluid and plasma biomarkers in Alzheimer disease. Nat Rev Neurol 2010;6:131-44.

89. Motta M, Imbesi R, Di Rosa M, et al. Altered plasma cytokine levels in Alzheimer's disease: correlation with the disease progression. Immunol Lett 2007;114:46-51.

90. Brosseron F, Krauthausen M, Kummer M, et al. Body fluid cytokine levels in mild cognitive impairment and Alzheimer's disease: a comparative overview. Mol Neurobiol 2014;50:534-44.

91. Aisen PS, Schafer KA, Grundman M, et al. Effects of rofecoxib or naproxen vs placebo on Alzheimer disease progression: a randomized controlled trial. JAMA 2003;289:2819-26.

92. Reines SA, Block GA, Morris JC, et al. Rofecoxib: no effect on Alzheimer's disease in a 1-year, randomized, blinded, controlled study. Neurology 2004;62:66-71.

93. Group ADC, Bentham P, Gray R, et al. Aspirin in Alzheimer's disease (AD2000): a randomised open-label trial. Lancet Neurol 2008;7:41-9.

94. Rogers J, Kirby LC, Hempelman SR, et al. Clinical trial of indomethacin in Alzheimer's disease. Neurology 1993;43:1609-11.

95. Breitner JC, Baker LD, Montine TJ, et al. Extended results of the Alzheimer's disease anti-inflammatory prevention trial. Alzheimers Dement 2011;7:402-11.

96. Gorlovoy P, Larionov S, Pham TT, et al. Accumulation of tau induced in neurites by microglial proinflammatory mediators. FASEB J 2009;23:2502-13.

97. Yamanaka M, Ishikawa T, Griep A, et al. PPARgamma/ RXRalpha-induced and CD36-mediated microglial amyloid-beta phagocytosis results in cognitive improvement in amyloid precursor protein/presenilin 1 mice. J Neurosci 2012;32:17321-31.

98. Heneka MT, Carson MJ, El Khoury J, et al. Neuroinflammation in Alzheimer's disease. Lancet Neurol 2015;14:388-405. 